\title{
Miniaturized ultra-wideband coplanarwaveguide lowpass filter with extended stop band
}

\author{
Elmahjouby Sghir ${ }^{1}$, Ahmed Errkik ${ }^{2}$, Jamal Zbitou $^{3}$, Otman Oulhaj ${ }^{4}$, Ahmed Lakhssassi ${ }^{5}$, \\ Mohamed Latrach ${ }^{6}$ \\ ${ }^{1,2,3}$ LMEET Laboratory FST of Settat Hassan 1st University, Morocco \\ ${ }^{4} \mathrm{FP}$ of Larache, Abdelmalek Essaâdi University, Morocco \\ ${ }^{5}$ University of Quebec in Outaouais, Canada \\ ${ }^{6}$ Microwave group ESEO Angers France, France
}

\section{Article Info \\ Article history: \\ Keywords: \\ CPW \\ Lowpass filter \\ Miniaturized \\ UWB}

Received Jan 5, 2020

Revised Mar 28, 2020

Accepted Apr 12, 2020

\author{
Corresponding Author: \\ Elmahjouby Sghir, \\ Department of Physics, \\ University of Hassan 1st Morocco, \\ Casablanca 20000, Morocco. \\ Email: elmahjouby.sghir@gmail.com
}

\begin{abstract}
In this article, we propose a novel design of large rejected band of miniaturized ultra wide band (UWB) of a planar CPW low pass filter "LPF" based on the use of periodic elements of 'e' slots. The goal of this work is to develop a new structure of Low Pass Filter with the following criterion: Miniature, Compact and Easy for Fabrication. The Miniaturization of this structure is achieved by entering the 'e' slot in etching area in the ground of CPW line, to save the standard gap of the adapted coplanar line. The designed coplanar LPF is a compact filter having a large band pass and extended stop band, with the possibility to associate easily with others RF and microwave planar circuits. The entire area of the proposed structure of CPW LPF is $14.3 \times 20 \mathrm{~mm} 2$.
\end{abstract}

Copyright (C) 2020 Institute of Advanced Engineering and Science. All rights reserved.

\section{INTRODUCTION}

Periodic structures of various kinds have been the driving force behind the interest researchers toward miniaturizing of structures. The miniaturized filters with high and low insertion-loss are important requirements in RF and microwave applications $[1,2]$. In literature we can find a few approaches address to design such this filter miniaturization, among which are the use of open stubs [3], lumped-element filters [4], stepped impedance [5], high temperature superconducting (HTS) filters, bulk acoustic-wave (BAW) filters, and slow-wave distributed resonator filters [6-8].

Using the two sides of the ground provides additional miniaturization for structure. In both free etched area $[9,10]$, witch here a rectangle DGS and loaded DGS $[11,12]$, by entering the 'e' slot in etching area in the ground of CPW line, an electric and magnetic coupling can be implemented, which allows for an intelligible design of simple an miniaturized structures, such as this proposed low pass filter, and many other structures with the same procedure.

In order to reach an accommodation between size and performance, both DGS with different configurations and slot lines, together inserted in coplanar waveguides lines (CPWs) are other important procedure for the realization of resonators and filters for microwave and millimeter-waves circuits [13-15]. Some compact structures based on periodic structures of DGS configuration and other based on periodic slots have been proposed to construct a slow-wave transmission line and has been used in the design of a miniature low-pass filter $[16,17]$. This paper translates our efforts to design and develop a miniaturized ultra wide band 
(UWB) of a planar CPW LPF and extended stop band with an ideal slow-wave structure, low loss properties, and easy fabrication.

\section{FILTER DESIGN AND CONFIGURATION}

Figures 1 show the simple proposed 'e' slot connected to conductor line of coplanar waveguide with width wc of $1.5 \mathrm{~mm}$ and the gap $\mathrm{G}$ of $0.4 \mathrm{~mm}$. This topology is placed on the two sides of cpw, and it is excited by $50 \Omega$ line. The proposed structure is coupled to metallic ground plane through a dielectric substrate with $\varepsilon r=4.4$ and thickness $\mathrm{h}=1.6 \mathrm{~mm}$. The investigated ' $\mathrm{e}$ ' slot consists of four slices with different length, which are connected to the conductor line. The 'e' slot corresponds to an equivalent capacitance and inductance. All the dimensions of the proposed structure are depicted in Table 1.

The filter design is started with a simple CPW line, passing to a rectangular DGS (with dimensions are: $\mathrm{Lr}=1 \mathrm{r}=3 \mathrm{~mm}$ ) in the ground, in which we have inserted the 'e' slot as shown in Figure 1.

Figure 2 shows the simulation results of the proposed configuration with one unit of 'e' slot, as we can conclude, it is clear that the filter provides low pass band behavior, with a cutoff frequency equal to 7 $\mathrm{GHz}$ corresponding to a reflection coefficient around $-10 \mathrm{~dB}$ in the whole bandwidth with very low and flat insertion loss less than $-0.5 \mathrm{~dB}$. From the S-parameter responses, the suppression is inadequate because limit on the separation between the insertion loss and return loss in the game frequency between 6-10 GHz.

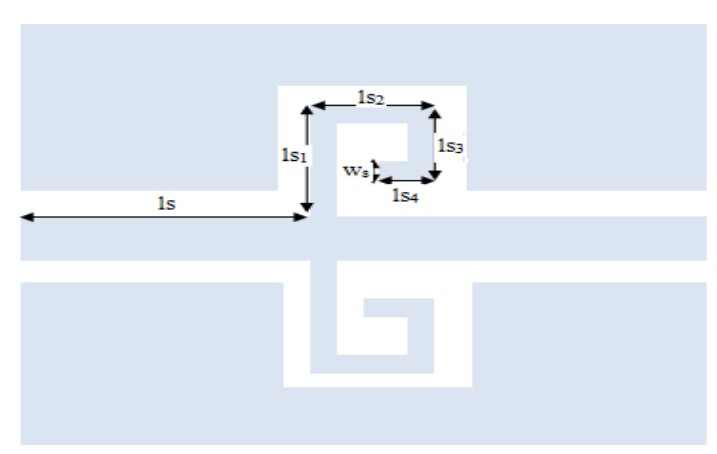

Figure 1. Structure of CPW LPF with one unit of 'e' slot

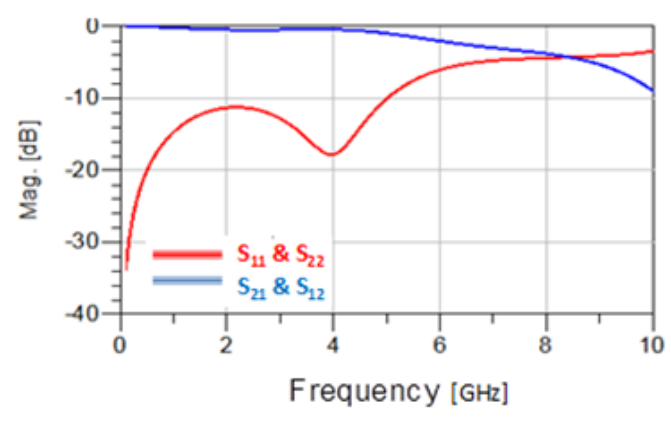

Figure 2. S-parameters versus frequency of the designed LPF filter

Table 1. Values of the different parameters of the bandpass filter

\begin{tabular}{cc}
\hline Parameters & Value $(\mathrm{mm})$ \\
\hline L & 20 \\
W & 14.3 \\
lr & 3 \\
Lr & 3 \\
Ws & 0.5 \\
ls & 9 \\
ls1 & 2.92 \\
ls2 & 2 \\
ls3 & 1.5 \\
ls4 & 1 \\
ds & 3.6835 \\
d1 & 4.3165 \\
d2 & 1.1835 \\
\hline
\end{tabular}

\section{PROPOSED DEVICE OF LPF}

In an attempt to improve the performance of S-parameters and the rejection band characteristics, the technique of the periodic cells is a good candidate. In the research, augmentation the number of unit cells and adjusting the distance between cells influence the response of S-parameters characteristics [18-26]. So to adjust the S-parameters in terms of suppression, rejection band and stop band, an optimized 'e' slot structure inserted and repeated along the two sides of conductor line. Therefore, the proposed structure of CPW LPF validated into simulation after many optimizations steps using Momentum integrated in ADS. Figure 3 depicts the schematic of the proposed structure with periodic cells. 
The parameters of the initial low pass filter with one unit cell are kept unchanged, while two other symmetrical 'e'slots are introduced and separate with $\mathrm{ds}=3.6835 \mathrm{~mm}$ distance as shown in Figure 3 . Figure 4 shows the simulated S-parameters of the proposed device of low pass filter with three 'e' slots Cells. As a result, the cascaded LPF with just three periodic cells present a good suppression performance and a sharp roll off with improved stop band. The return loss in pass band is greater than $13 \mathrm{~dB}$, with no more than 0.52 $\mathrm{dB}$ ripple level in the pass band, while the stop band rejection level going up $50 \mathrm{~dB}$ with wide stopband.

By taking the final dimensions of structure, and to ensure the validity of our proposed filter, the simulation result is given by ADS momentum, HFSS and CST Microwave Studio in order to compare the results and to give more credibility for the proposed structure. The s-parameters results show good agreement between ADS momentum, HFSS and CST Microwave Studio in the performance of insertion loss and return loss, and the results with the three simulators are shown in Figure 5.

Figure 6 presents the phase simulation of insertion loss (S21) and return loss (S11). As we shown in Figures, the proposed structure of low pass filter accepted a linear phase for the insertion loss and return loss for the ultra wide band UWB applications from $0.1 \mathrm{GHz}$ to $10 \mathrm{GHz}$. As results depicts, we can see that the proposed filter with three periodic cells of ' $\mathrm{e}$ ' slot meet the requirement.

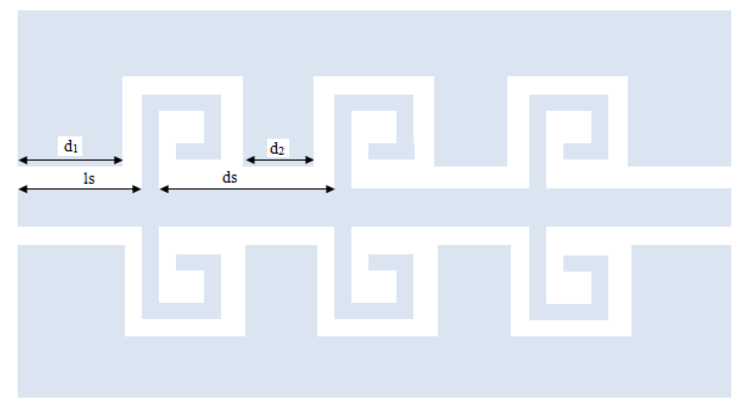

Figure 3. The proposed device of low pass filter with cascaded 'e' slots Cells

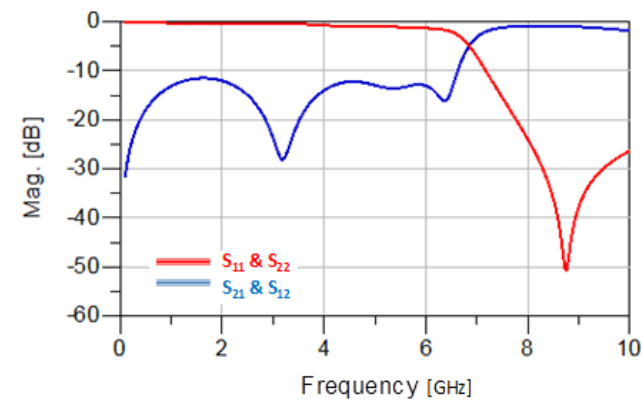

Figure 4. Simulated S-parameter of the proposed LPF with periodic cells device

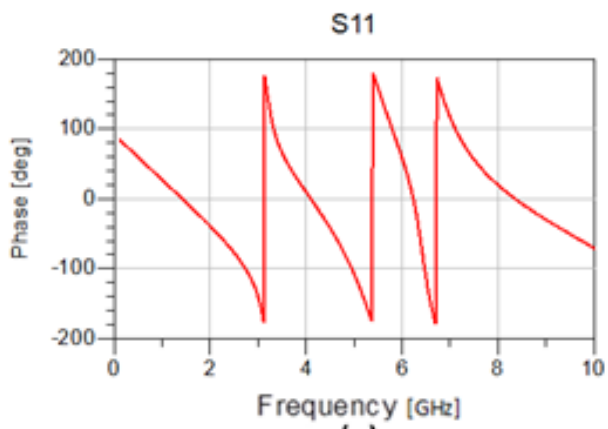

(a)

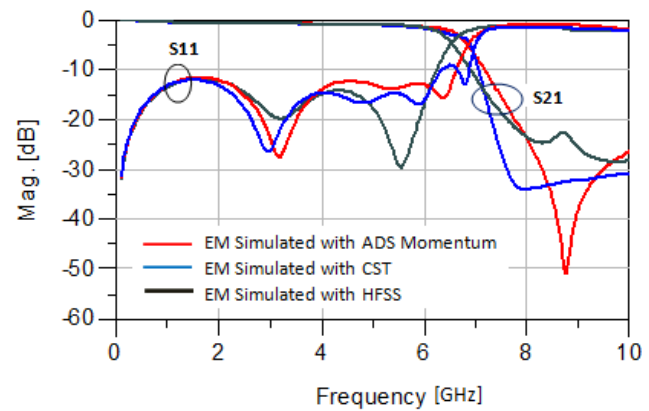

Figure 5. Comparison results between different methods of simulation

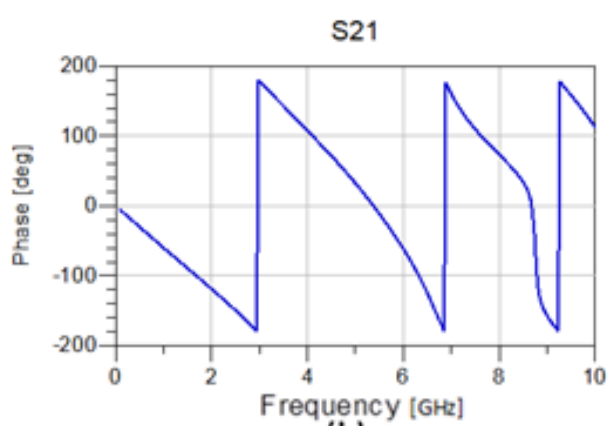

(b)

Figure 6. Simulation phase responses of low pass filter designed: (a) of return loss, (b) of insertion loss 


\section{DISTRIBUTION OF SURFACE CURRENT AT PASS-BAND AND STOP-BAND FREQUENCY RANGES}

Figures 7(a) and 7(b) show the current density distributions in the passband and in the stopband, respectively. The power was fully transmitted along the proposed filter, from the input to the output, at the frequency of $3.5 \mathrm{GHz}$, which means that the filter is in a good passband state. In the other side, in the filter stopband at the $8.5 \mathrm{GHz}$ frequency, there is a high current distribution energies in the first part of structure, while fully attenuated, since no current near to the output port 2 , which means that the filter is in strong stopband state. This experiment results demonstrate the relationship and the good reliance between EMsimulation results and the current energies distribution along the proposed structure. All these investigations prove that our proposed low pass filter with just three periodic cells of 'e' slot has good performances.

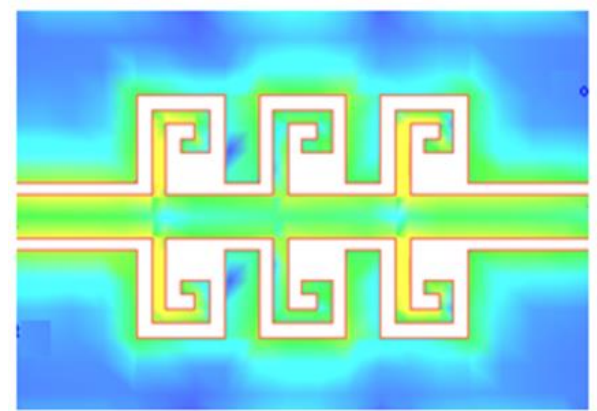

(a)

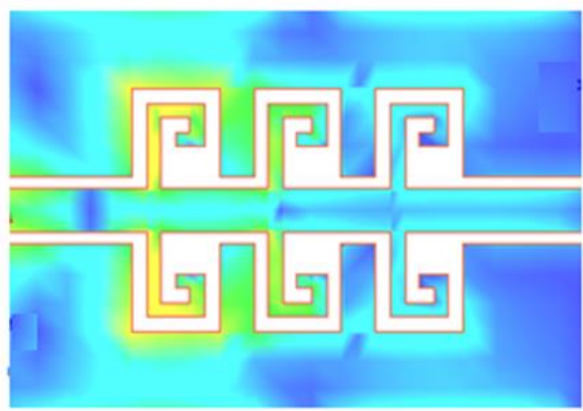

(b)

Figure 7. Simulated surface current density. (a) at $\mathrm{f}=3.5 \mathrm{GHz}$, (b) at $\mathrm{f}=8.5 \mathrm{GHz}$.

\section{CONCLUSION}

This paper comes with a miniaturized and several novel periodic structures for a CPW waveguide. The periodic structures based on three 'e' slots series cascade offer very easy fabrication, very low insertion loss, and simple filter synthesis. A periodic series with the proposed configuration results in miniature low-pass filters that offer high attenuation levels in the stopband while reducing filter area size compared with other standard structure. This novel periodic structure is potential to be used in RF and microwave integrated circuits.

\section{ACKNOWLEDGEMENTS}

We thank Mr. Mohamed Latrach from ESEO Angers in France, for allowing us to use and to perform simulations by using electromagnetic solvers and measurement instruments.

\section{REFERENCES}

[1] Rahimian A., "Modeling and Performance of Microwave and Millimeter-Wave Layered Waveguide Filters," TELKOMNIKA Indonesian Journal of Electrical Engineering, vol. 11, no. 7, pp. 3523-3533, 2013.

[2] Hsieh L. H. and Chang K., "Compact elliptic-function low-pass filters using microstrip stepped-impedance hairpin resonators," IEEE Transactions on Microwave Theory and Techniques, vol. 51, no. 1, pp. 193-199, 2003.

[3] Pingjuan Z., Minquan L., "A Novel Compact Microstrip Lowpass Filter with Sharp Transition and Improved Stopband," Indonesian Journal of Electrical Engineering and Computer Science (IJEECS), vol. 13, no. 1, pp. 85-90, 2015.

[4] Matthaei G. L., et al., "Microwave Filters, Impedance Matching Networks and Coupling Structures," Artech Microwave Library, 1980.

[5] Hsieh L. H. and Chang K., "Compact lowpass filter using stepped impedance hairpin resonator," Electronics Letters, vol. 37, 14, pp. 899-900, 2001.

[6] Reza Azadegan, Kamal Sarabandi., "Miniature High-Q Double-Spiral Slot-Line Resonator Filters," IEEE Transactions on Microwave Theory and Techniques, vol. 52, no. 5, 2004.

[7] R. Levy, R. V. Snyder, and G. L. Matthaei., "Design of microwave filters," IEEE Trans. Microwave Theory Tech, vol. 50, pp. 783-793, Mar 2002.

[8] P. Bradley, R. Ruby, J. D. Larson, Y. Oshmyansky, D. Figueredo., "A film bulk acoustic resonator (FBAR) duplexer for USPCS handset applications," in Proc. IEEE MTT-S Int. Microwave Symp. Dig, vol. 1, pp. 367-370, 2001. 
[9] E. Sghir, A. Errkik, J. Zbitou et al., "A novel compact CPW tunable stop band filter using a new Z-DGS-resonator for microwave applications," TELKOMNIKA, Vol. 17, No. 5, pp. 2410- 2417, Octobre 2017.

[10] A. Boutejdar, M. Amzi, S. Bennani., "Design and Improvement of a Compact Bandpass Filter using DGS Technique for WLAN and WiMAX Applications," TELKOMNIKA, Vol. 15, No. 3, pp. 1137- 1144, September 2017.

[11] E. Sghir, A. Errkik, J. Zbitou et al., "A novel compact CPW band-stop filter using O-DGS configuration," International Journal of Electrical and Computer Engineering (IJECE), vol. 9, no. 6, pp. 4836- 4844, 2019.

[12] M. Yang, J. Xu, Q. Zhao, L. Peng, and G. Li., "Compact, broad-stopband lowpass filters using sirs-loaded circular hairpin resonators," Progress In Electromagnetics Research, vol. 102, pp. 95- 106, 2010.

[13] K. C. Gupta, P. Garg, I. Bahl, and P. Bhartia., "Microstrip Lines and Slotlines," 2nd ed. Norwood, MA, Artech House, 1996.

[14] J. K. A. Everard and K. K. M. Cheng., "High-performance direct coupled bandpass filter on coplanar waveguide," IEEE Trans. Microwave Theory Tech, vol. 41, pp. 1568-1573, 1993.

[15] Elmahjouby Sghir, Ahmed Errkik, Jamal Zbitou et al., "A Novel Compact CPW Multi-StopBand Filter with DGS Integrating Circular Ring Resonator," Transactions on Machine Learning and Artificial Intelligence, vol. 5, no. 4, pp. 677-682, 2017.

[16] Elmahjouby Sghir, Ahmed Errkik, Jamal Zbitou, A.Tajmouati, Larbi El Abdellaoui, Mohamed Latrach., "A Novel Compact CPW LowPass Filter Integrating Periodic Triangle DGS Cells," Transactions on Machine Learning and Artificial Intelligence, vol. 5, no. 4, pp. 457-462, 2017.

[17] J. Sor, Y. Qian, and T. Itoh., "Miniature low-loss CPW periodic structures for filter applications, IEEE Trans. Microwave Theory Tech, vol. 49, pp. 2336-2341, 2001.

[18] J. Y. Wu, Y. H. Tseng and W. H. Tu., "Design of compact lowpass filter with ultrawide stopband using thin slots," Progress In Electromagnetics Research, vol. 31, pp. 137-151, 2012.

[19] J. W. No and H. Y. Hwang., "A design of cascaded CPW lowpass filter with broad stopband," IEEE Microwave and Wireless Components Letters, vol. 17, pp. 427-429, 2007.

[20] C. HanJan, H. TsungHui, and C. ChinSheng et al., "A novel crossshape DGS applied to design ultrawide stopband lowpass filters," IEEE Microwave and Wireless Components Letters, vol. 16, no. 5, pp. 252-254, 2006.

[21] M. Kazerooni, N. P. Gandji, A. Cheldavi, and M. Kamarei., "A new microwave bandstop filter using defected microstrip structure (DMS)," PIERS Proceedings, Moscow, pp. 697- 700, 2009.

[22] A. Boutejdar, G. Nadim, S. Amari, and A. S. Omar., "Control of bandstop response of cascaded microstrip lowpass-bandstop filters using arrowhead slots in backside metallic ground plane," Proc. IEEE AP-Symp, 2005.

[23] M. Nosrati, T. Faraji, and Z. Atlasbaf., "A compact composite broad stop-band elliptic function low-pass filter for ultra-wideband applications using interdigital capacitors," Progress In Electromagnetics Research Letters, vol. 7, pp. 87-95, 2009.

[24] J. Y. Wu, Y. H. Tseng and W. H. Tu., "Design of compact lowpass filter with ultraרwide stopband using thin slots," Progress In Electromagnetics Research C, vol. 31, pp. 137- 151, 2012.

[25] J. W. No and H. Y. Hwang., " A design of cascaded CPW low pass filter with broad stopband," IEEE Microwave and Wireless Components Letters, vol. 17, pp. 427- 429, 2007.

[26] C. Han Jan, H. Tsung Hui, and C. Chin Sheng et al., "A novel cross-shape DGS applied to design ultra-wide stopband low-pass filters," IEEE Microwave and Wireless Components Letters, vol. 16, no. 5, pp. 252- 254, 2006. 